\title{
Association and Interaction Between Dietary Patterns and Gene Polymorphisms in Liangshan Residents with Hyperuricemia
}

Tingting Li

Southwest Medical University, Luzhou

Shuangjing Li

Ya an Vocational and Technical College, Yaan

Tian Tian

Southwest Medical University, Luzhou

Zhichao Nie

Southwest Medical University, Luzhou

Wangdong Xu

Southwest Medical University, Luzhou

Longjian Liu

Drexel University

Hong Jia ( $\nabla$ jhong_Iz@163.com )

Southwest Medical University, Luzhou

\section{Research Article}

Keywords: dietary pattern, hyperuricemia, gene, interaction, factor analysis

Posted Date: June 30th, 2021

DOl: https://doi.org/10.21203/rs.3.rs-620530/v1

License: (c) (i) This work is licensed under a Creative Commons Attribution 4.0 International License. Read Full License 


\section{Association and Interaction Between Dietary Patterns and Gene}

\section{Polymorphisms in Liangshan Residents with Hyperuricemia}

3 Tingting $L i^{1}$, Shuangjing $L i^{2}$, Tian Tian ${ }^{1}$, Zhichao Nie ${ }^{1}$, Wangdong $X u^{l}$, Longjian Liu ${ }^{3}$,

$4 \quad$ Hong $\mathrm{Jia}^{1, *}$

$5 \quad{ }^{1}$ School of Public Health, Southwest Medical University, Luzhou, China; ${ }^{2}$ Nursing Department, Ya

6 an Vocational and Technical College, Yaan, China; ${ }^{3}$ Department of Epidemiology and Biostatistics,

7 School of Public Health, Drexel University, United States

$8 \quad{ }^{*}$ Corresponding author: Hong Jia, E-mail address: jhong_1z@163.com

9 Telephone number: 0830-2985332 


\section{ABSTRACT}

Background: Hyperuricemia (HUA) is caused by genetic and dietary factors. However, little is known about the ways in which diet and gene interactions influence HUA or the role of dietary patterns in HUA.

Objectives: We aimed to explore the association between dietary patterns and HUA as well as dietary patterns and gene interactions in HUA among the Yi ethnic group of China.

Methods: Food consumption information was collected using a semi-quantitative food frequency questionnaire. Vein blood samples were collected after overnight fasting from all participants and DNA was extracted from peripheral blood leukocytes. Dietary patterns were derived using factor analysis.

Results: The study included 2646 participants aged 18-88 years with a HUA prevalence of $26.8 \%$. Three major dietary patterns were identified and shown to be associated with HUA prevalence, with meat-based diets having a higher association with HUA than plant-based or regionally specific diets. The frequency of the T allele at $A B C G 2$ rs2231142 and SLC2A9 rs11722228 loci was higher in HUA than in non-HUA populations. An additive interaction between a meat-based diet and the rs2231142 locus was associated with the risk of HUA. The relative excess risks of interaction (RERI), attributable proportion of interaction (AP), and synergy index (S) were 0.482 (95\% CI $=0.012,0.976), 0.203(95 \% \mathrm{CI}=0.033,0.374), 1.544(95 \% \mathrm{CI}=1.012,2.355)$, respectively.

Conclusions: The meat-based dietary pattern was associated with an increased risk of 
HUA. There was an additive interaction between a meat-based dietary pattern and the $A B C G 2$ rs2231142 locus in study participants. Individuals with the rs2231142 $\mathrm{T}$ allele had a higher frequency of HUA than those with the rs2231142 GG allele. Individuals with the rs2231142 $\mathrm{T}$ allele but a healthy dietary pattern could have a reduced HUA risk.

Keywords: dietary pattern; hyperuricemia; gene; interaction; factor analysis

\section{Introduction}

Hyperuricemia (HUA) is a purine metabolic disorder. The most well-known disease induced by HUA is gout, but many studies have reported that HUA also plays important roles in cardiac-kidney-vascular system diseases and metabolic syndromes (Mets) [1]. A meta-analysis in 2015 reported that the pooled prevalence of HUA is $13.3 \%$ in China [2]. A national survey in the US found that the prevalence of HUA is substantial, with $20.2 \%$ in males and $20.0 \%$ in females aged 20 years and older [3]. HUA has become the second most common metabolic disease after diabetes mellitus [1].

The known risk factors for developing HUA include genetic and environmental factors as well as interactions between them [1]. Recent genome-wide association studies (GWAS) have identified significant associations between single nucleotide polymorphisms (SNPs) in $A B C G 2$ and $S L C 2 A 9$ in HUA cases [4]. Studies have shown that a mutation at the rs 2231142 locus of $A B C G 2$ can cause a $53 \%$ reduction in $A B C G 2$ mediated serum uric acid (SUA) transport [5], and that a variation at the rs 11722228 
locus of SLC2A9 can also affect the transport and reabsorption of SUA [6].

Along with inherited genetic variants, specific dietary components also play a significant role in the development of HUA [7]. Previous studies have reported that red meat, seafood, and alcohol consumption are associated with the risk of HUA $[8,9]$. However, most of the existing research focuses on the effects of a single food or nutrient rather than the interaction between multiple food items, which may be more relevant to the study of disease association $[10,11]$. The dietary pattern method uses complex combinations of foods and nutrients to investigate the association between multiple food items and disease [12]. A cross-sectional study found that the 'animal and fried foods' pattern was associated with a higher prevalence of HUA [13], while another study demonstrated that there was no significant association between dietary patterns and HUA in Chinese participants [11]. Hence, the association between dietary patterns and HUA remains unclear.

Potential gene-environment interactions are key features in the development of complex diseases, including HUA and gout [14]. Evidence has suggested that the intake of sugar-sweetened beverages and SLC2A9 variants interact in the pathogenesis of gout [15]. However, there are few studies on diet and gene interaction in HUA [7,14-16] and to our knowledge, no previous study has examined the relationship of dietary patterns and gene interaction in HUA risk in the Chinese population.

The Liangshan Yi Autonomous Prefecture is located in southwestern Sichuan province, where the aboriginal Yi people maintain a unique dietary culture and customs [17]. Our previous study found that the prevalence of HUA among adult Yi people was 
$22 \%$, which was significantly higher than that in other areas of China [18]. Therefore, we aimed to identify their major dietary patterns and assess the association between dietary patterns and the risk of HUA among adult Yi people in China. We also explore the effects of dietary patterns and gene interactions on HUA.

\section{Methods}

\section{Participants}

A cross-sectional study was conducted on the Yi people located in the Liangshan District, Sichuan Province, China, from July 2014 to February 2016. A representative sample was obtained using a multistage stratified cluster sampling method. Details of the study design were described in our previous study [19]. We excluded participants who had unretained blood samples or dietary questionnaires that were more than $50 \%$ incomplete. Of the 3188 participants, 2646 were included in the analyses. The protocol of this study was approved by the Ethics of Research Committee of Southwest Medical University. All methods were performed in accordance with the relevant guidelines and regulations of the Ethics of Research Committee of Southwest Medical University. Informed consent was obtained from all participants.

\section{Questionnaire survey}

The on-site survey was conducted by well-trained investigators through face-toface interviews. Participants were asked to complete a questionnaire that included demographic and lifestyle information. Their dietary intake was assessed using a semi- 
quantitative 52-item food frequency questionnaire (FFQ) that inquired about the types of food items, frequency (daily, weekly, monthly, yearly, or never), and amount of food consumed over the past year (serving size per gram). The FFQ was designed based on culture-specific dishes and tested on local samples to check its applicability under the Yi culture. Alcohol consumption was calculated based on the drinking frequency and consumption of different alcoholic beverages.

\section{Physical examination}

Height and weight were measured by trained professionals to the nearest $0.1 \mathrm{~cm}$ and $0.01 \mathrm{~kg}$, respectively. Body mass index (BMI) was calculated by weight/height ${ }^{2}$ $\left(\mathrm{kg} / \mathrm{m}^{2}\right)$. Blood pressure (BP) was measured twice from the upper left arm after $5 \mathrm{~min}$ rest in a seated position. Waist circumference was measured with a non-retractable tape measure to the nearest $0.1 \mathrm{~cm}$ at the umbilical level with the participants standing and breathing normally. Hip circumference was measured to the nearest $0.1 \mathrm{~cm}$ around the symphysis pubis and the posterior gluteus maximus.

\section{Assessment of hyperuricemia and other variables}

HUA was defined as serum uric acid (SUA) concentrations $>420 \mu \mathrm{mol} / \mathrm{L}(7.0$ $\mathrm{mg} / \mathrm{dL})$ for males and $>360 \mu \mathrm{mol} / \mathrm{L}(6.0 \mathrm{mg} / \mathrm{dL})$ for females [1]. Overnight fasting blood samples were collected from each participant. SUA, triglycerides (TG), total cholesterol (TC), fasting plasma glucose (GLU), high-density lipoprotein cholesterol (HDL-C), and low-density lipoprotein cholesterol (LDL-C) were measured using an 
automatic biochemical analyzer (Mindary, BS-820, Shenzhen, China).

\section{Genomic DNA extraction and genotyping}

Genomic DNA was extracted from peripheral blood leukocytes and a Nano Drop 2000 ultraviolet spectrophotometer was used to measure its quantity and quality. Genotyping was performed by Kompetitive Allele Specific PCR (KASP). The detection success rates of the $A B C G 2$ rs 2231142 and SLC2A9 rs 11722228 loci were $99 \%$ and 97.2\%, respectively. The SNPs were selected based on the following criteria: 1) SNP with $\mathrm{P}<1.0 \times 10-5$ for all GWAS samples; 2) when multiple SNPs had a strong LD $\left(\mathrm{r}^{2} \geq 0.8\right)$, SNPs previously reported in the literature were used prior to selection; 3 ) clear genotyping clusters; and 4) Minor Allele Frequency(MAF) $\geq 0.05$. Based on quality control criteria, SNPs were excluded when the $\mathrm{P}<0.001$ for the Hardy-Weinberg equilibrium (HWE) test, $\mathrm{MAF}<0.01$, or genotype call rate $<95 \%$.

\section{Identification of dietary patterns}

According to the Food Composition Table, similarity of nutrient profiles, and purine contents, we collapsed 52 food items into 17 food groups. The Kaiser-Meyer-Olkin (KMO) measure of sampling adequacy and Bartlett's sphere test were used to evaluate the adequacy of correlation matrices with the data. Principal component factor analysis (PCFA) was applied to derive dietary patterns. Moreover, varimax rotation was applied for greater interpretability. After evaluation of eigenvalues $>1$, the cumulative contribution rate, and the scree plot, three factors were determined. Food items with a 
factor loading $>0.30$ (absolute value) were regarded as the main components of each pattern. The higher the factor loading of a food group, the greater the contribution of that group to the pattern. The labeling of dietary patterns was based on the interpretation of foods with high factor loadings for each pattern [20]. Dietary pattern scores were calculated by summing the product of the standardized intake for each group multiplied by the regression coefficients before they were categorized into quartiles (Qs).

\section{Statistical analyses}

The factor scores of each dietary pattern were divided into quartiles with Q4 and Q1 the highest and lowest categories for the intake of each dietary pattern, respectively. The association between the quartile categories of dietary pattern scores and HUA was examined using logistic regression analysis. Data for continuous variables are presented as mean \pm standard deviation. Categorical variables were generally reported as the sum (percentage) and analyzed using a chi-square test. The Kaiser-Meyer-Olkin(KMO) measure of sampling adequacy $(0.727)$ and Bartlett's test of sphericity $(\mathrm{P}<0.001)$ showed that the factor model as a whole was significant. The additive interaction was measured using the Delta method described by Rothman et al. [21], while the parameter estimates and covariance matrix of the logistic regression model were substituted in the Excel program developed by Andersson et al. [22] to calculate the additive interaction index. The indicators used for evaluating the additive interaction included the excess relative risk (RERI), attribution ratio (AP), and interaction index (S). If the 95\% CI of RERI and AP were $>0$, and the $95 \% \mathrm{CI}$ of $\mathrm{S}>1$, there was a synergistic interaction 
between the dietary pattern and the gene in HUA. Data were analyzed using SPSS software (SPSS Inc. Version 24). All P values were two-tailed, and the difference was considered significant when $\mathrm{P}<005$.

\section{Results}

\section{Participants}

A total of 2646 subjects were included in this study, including 1445 males and 1201 females. The prevalence of HUA was $26.8 \%$ in all participants, but $34.9 \%$ in males and $17.2 \%$ in females. Participants with and without HUA showed significant differences in age, gender, ethnicity, education, and occupation. Compared with non-HUA participants, patients with HUA showed higher expression of SUA, TC, and TG, as well as higher WC, WHR, BMI, and BP (Table 1). The participant flowchart is shown in Figure 1.

\section{Extraction of dietary patterns}

Three major dietary patterns were identified by factor analysis in the tested population (Table 2). Factor 1 was defined as the 'meat-based' pattern and characterized by a high intake of animal organs, seafood, fresh meat, and eggs; factor 2 was characterized as a 'plant-based' pattern and included the intake of mushroom algae, beans and their products, nuts, and fruits; and factor 3, was the 'local special' pattern was characterized by the intake of marinated smoked meat and grease. These three patterns accounted for $33 \cdot 87 \%$ of total variance, with the 'meat-based,' 'plant- 
based,' and 'local special' dietary patterns constituting 12.64\%, $12.54 \%$, and $8.69 \%$ of the total variance, respectively.

\section{Association between dietary patterns and HUA}

As shown in Table 3, there was a significant association between the 'meat-based' pattern and a high prevalence of HUA. Compared with the participants in the lowest quartile, the OR for the highest quartile was $1.878(95 \% \mathrm{CI}=1.461,2.415, P<0001)$ and $1.385(95 \% \mathrm{CI}=1.052,1.823, P<005)$ before and after adjustment for age, sex, and ethnicity, respectively. The other two dietary patterns were not related to the prevalence of HUA in the participant population after adjustment.

\section{Hardy-Weinberg equilibrium test and genotype frequency distribution}

The allele frequencies for both SNPs in the $A B C G 2$ case group $\left(\chi^{2}=0156, P=0.925\right)$, control group ( $\left.\chi^{2}=0.303, P=0.859\right), S L C 2 A 9$ case group $\left(\chi^{2}=0.156, P=0.925\right)$, and control group ( $\left.\chi^{2}=0.683, P=0.711\right)$ were in Hardy-Weinberg equilibrium $(P>0.05)$. The results reveal that the population used in the present study exhibited adequate group representation. Table 4 displays the genotype frequency distribution of the $A B C G 2$ and SLC2A9 genes. For both loci, the T allele in the HUA group was higher than that in the non-HUA group.

\section{Different genotypes and HUA}

As shown in Figure 2, when the GG wild-type genotype at the rs2231142 locus in 
$A B C G 2$ was used as a reference, the risk of HUA increased with the number of T alleles.

Additionally, compared with the GG wild-type genotype, the risk of HUA in individuals with GT and TT mutant genotypes was 1.784 times $(95 \% \mathrm{CI}=1.471,2.163)$ and 2.993 times $(95 \% \mathrm{CI}=2.015,4.445)$, respectively. After adjustment for age, sex, and ethnicity, the association between the rs2231142 locus and HUA was still significant $(P<0.001)$. with the CC wild-type genotype, the $\mathrm{CT}$ and TT mutant genotypes increased the risk of HUA by $22.5 \%(\mathrm{OR}: 1.225,95 \% \mathrm{CI}=1.015,1.478)$ and $39.2 \%(\mathrm{OR}: 1.392,95 \% \mathrm{CI}=$ $1.029,1.884)$, after adjustment for confounders, respectively.

\section{Interaction analysis between dietary patterns and genetics}

There was no evidence that the three dietary patterns had multiplication interaction

effects on HUA risk in combination with the $A B C G 2$ rs2231142 and SLC2A9 rs11722228 loci. Therefore, we next examined whether there were additive dietary and genetic interactions associated with HUA in the tested population.

The three dietary pattern factor scores were divided into two quantiles, with the low quantile as the reference and the rs2231142 locus referenced with the GG wild-type genotype. There was an additive interaction between the pattern of 'meat-based' and the rs2231142 locus associated with the risk of HUA; the RERI, AP, and S were 0.482 $(95 \% \mathrm{CI}=0.012,0.976), 0.203(95 \% \mathrm{CI}=0.033,0.374)$, and $1.544(95 \% \mathrm{CI}=$ 
including 0 and the interaction coefficient $\mathrm{S}$ including 1 . Using the $\mathrm{CC}$ wild-type genotype as a reference, the results showed no additive interaction between the rs11722228 locus and the three dietary patterns.

When the average SUA levels of each genotype at the $A B C G 2$ rs2231142 locus was compared under the "animal diet pattern" quartile, that SUA levels increased with the number of $\mathrm{T}$ risk alleles $(\mathrm{GG} \rightarrow \mathrm{GT} \rightarrow \mathrm{TT})$. In addition, the SUA level increased as the "meat-based pattern" factor score increased in the same genotype (Figure 4).

\section{Discussion}

In this study, three major dietary patterns were identified in the Yi people of the Liangshan District, Sichuan Province, China that were mainly meat-based, plant-based, or associated with regionally specific cuisine. The main finding of this study is that the meat-based food pattern, which is characterized by high intake of animal organs, seafood, fresh meat, and eggs was positively associated with an elevated risk of HUA, whereas no significant association was found between the other two patterns and HUA risk.

To date, several studies have explored the association between diet and HUA. A cross-sectional study demonstrated that animal products and fried food patterns that are rich in pork, eggs, animal giblets, poultry, and fried wheat, was positively associated with a higher prevalence of HUA [13]. He et al. [23] found that a meat food pattern characterized by the intake of poultry, beef, processed and cooked meat, eggs, and fats, was associated with an elevated risk of HUA. In contrast, a study in Taiwan showed no significant association between any dietary pattern and HUA after adjustment [11]. 
Another study suggested that compared with individuals in the lowest quintile, those in the highest quintile of meat and seafood intake experienced increased risk of gout [8].

Our study found a positive association between the 'meat-based' dietary pattern and the prevalence of HUA, which is consistent with previous reports [13,23]. The mechanism that has been proposed to explain this connection is related to the high purine content of animal meat-based foods because excessive intake of purines may increase the level of SUA and thus, increase the risk of HUA [8]. Our previous study found that people in the Liangshan area are more likely to drink broth and the meat consumed is mainly fresh pork fat [17], which contains more purines and saturated fatty acids. The consumption of animal food products, especially the calories and fat in red meat, leads to centripetal obesity, which is a strong stimulus for increased plasma insulin levels that could lead to HUA $[13,24]$. A previous study found that a dietary pattern of soybean products and fruit was negatively associated with the prevalence of HUA [13]. Fruits and vegetables can provide rich dietary fiber and their antioxidant components have a protective effect against HUA [10]. However, we found no significant association between the other plant-based and regional dietary patterns and HUA in the present study, indicating that further research is still needed to clearly understand how diet patterns affect HUA prevalence.

The heritability of SUA concentration is approximately 40\%-70\% [25]. Previous GWAS studies have suggested an association between HUA susceptibility relative to dysfunctional $A B C G 2$ variants, with the rs2231142 locus being the most common variant [26]. In our study, we found that individuals with the $A B C G 2$ rs2231142 T allele 
had a higher frequency of HUA and SUA than those with the rs2231142 GG allele, which was consistent with results reported in the United States [27] and Mexico [28]. $A B C G 2$ is a high-capacity urate transporter that plays a key role in renal urate overload [26]. The risk of HUA that is attributed to $A B C G 2$ variants is $29.2 \%$, which is much higher than other typical environmental risks [29]. Our findings indicate that the rs2231142 locus variants of $A B C G 2$ are extremely important in HUA pathogenesis, consistent with the reported importance of $A B C G 2$ genotyping in the screening of HUA high-risk individuals [26].

The SLC2A9 gene encodes two GLUT9 isoforms of the class II facilitative glucose transport family [7]. A previous study reported that the rs 11722228 locus of SLC29 can explain $1.03 \%$ of the variation in SUA levels in the Chinese population [30]. Doring et al. reported that the most significant SNPs associated with SUA were within the SLC2A9 gene [31]. Our study also found that the SUA level in individuals with the rs11722228 locus showed increased instances of the T allele and was higher in the HUA population than in the non-HUA population.

Longitudinal studies have found that some individuals are more sensitive to unhealthy diets, reflecting the complex interaction between genetic factors and diet [14]. In this study, we found an additive interaction between a meat-based dietary pattern and the rs2231142 locus of $A B C G 2$. Under the same dietary pattern factor score, the increase of the T allele at the rs2231142 locus was associated with increased SUA levels. Moreover, individuals with this genotype also exhibited SUA levels that increased with the dietary pattern factor score. Similar to our results, Beydoun et al. [7] reported that 
12 SNP sites (including $A B C G 2$ and $S L C 2 A 9$ ) are closely related to SUA and found that there was a synergistic interaction between the genetic risk score (GRS) and red meat intake that increased the risk of increased SUA among women. In addition, a population-based study in Malaysia (32) showed that SUA concentration is affected by the interaction of a 'fruit diet pattern' and the VEGFR-2 rs2071559 locus, suggesting that fruit consumption may play a role in enhancing or suppressing the effects of VEGFR-2 polymorphisms. the hypothesis that different dietary intake can enhance or weaken the effect of genes population. Our study used a large sample size and careful study design to add new evidence of diet and genetic interactions on HUA prevalence in the Chinese population to the field. The findings of this study will provide new and necessary insight into HUA and assist in the development of intervention strategies. diseases such as Mets [33] and obesity [34]. Our study is among the few to explore the influence of dietary patterns and gene interactions on HUA. We also evaluated dietary patterns using FFQ, which consists of purine-containing foods and beverages commonly consumed by the Chinese population. This is important because dietary 
In addition, information from the questionnaires was collected by trained investigators, and thus our results are reliable.

This study had several limitations. First, it is a cross-sectional study and therefore cannot refer to causality. Second, although we adjusted for potential confounding variables in the multivariate adjusted model, we were unable to control for the effect of unmeasured confounding factors.

In conclusion, higher adherence to meat-based dietary pattern was associated with a higher prevalence of HUA in a Chinese population. There was a significant additive interaction between the meat-based dietary pattern and the $A B C G 2$ rs 2231142 locus on the risk of HUA in the Yi ethnic group of China. These findings may provide new insights into health policy and intervention strategies for controlling HUA among the Chinese population. Further prospective studies will be needed to validate our findings.

\section{Acknowledgements}

We would like to express our appreciation to the people who participated in this study for their cooperation and our support of the Liangshan Prefecture CDC, teachers, and students from Southwest Medical University, and Editage(www.editage.cn) for English language editing.

\section{Author contributions}

HJ: designed the research project; LJL: provided comments and critical review of the manuscript; TTL: analyzed the data and was primarily responsible for the final content 
342

343

344

345

346

347

348

349

350

351

352

353

354

355

356

357

358

359

360

361

362

of the manuscript. All authors wrote the paper and read and approved the final manuscript.

\section{Conflicts of interest}

The authors report no conflicts of interest.

\section{Funding}

This work was supported by the Danone Dietary Nutrition Research and Education Fund of Danone Institute (China) under grant number DIC2013-03.

\section{Data Share Statement}

Data described in the manuscript, code book, and analytic codes cannot be made available due to the University Institution Review Board requirement.

\section{References}

[1] Multidisciplinary Expert Task Force on Hyperuricemia and Related Diseases. Chinese Multidisciplinary Expert Consensus on the Diagnosis and Treatment of Hyperuricemia and Related Diseases. Chin Med J 2017;130:2473-2488. doi:10.4103/0366-6999.216416

[2] Liu R, Han C, Wu D, Xia X, Gu J, Guan H, et al. Prevalence of Hyperuricemia and Gout in Mainland China from 2000 to 2014: A Systematic Review and Meta-Analysis. Biomed Res Int 2015;2015:762820. doi:10.1155/2015/762820 
364

[3] Chen-Xu M, Yokose C, Rai SK, Pillinger MH, Choi HK. Contemporary Prevalence of Gout and Hyperuricemia in the United States and Decadal Trends: The National Health and Nutrition Examination Survey, 2007-2016. Arthritis Rheumatol 2019;71:991-999. doi:10.1002/art.40807

[4] George RL, Keenan RT. Genetics of hyperuricemia and gout: implications for the present and future. Curr Rheumatol Rep 2013;15:309. doi:10.1007/s11926-012-03098

[5] Woodward OM, Köttgen A, Coresh J, Boerwinkle E, Guggino WB, Köttgen M. Identification of a urate transporter, ABCG2, with a common functional polymorphism causing gout. Proc Natl Acad Sci USA 2009;106:10338-10342. doi:10.1073/pnas.0901249106

[6] Witkowska K, Smith KM, Yao SY, Ng AM, O'Neill D, Karpinski E, et al. Human SLC2A9a and SLC2A9b isoforms mediate electrogenic transport of urate with different characteristics in the presence of hexoses. Am J Physiol Renal Physiol 2012;303:F527F539. doi:10.1152/ajprenal.00134.2012

[7] Beydoun MA, Canas JA, Fanelli-Kuczmarski MT, Tajuddin SM, Evans MK, Zonderman AB. Genetic risk scores, sex and dietary factors interact to alter serum uric acid trajectory among African-American urban adults. Br J Nutr 2017;117:686-697. doi:10.1017/S0007114517000411

[8] Choi HK, Atkinson K, Karlson EW, Willett W, Curhan G. Purine-rich foods, dairy and protein intake, and the risk of gout in men. N Engl J Med 2004;350:1093-1103. doi:10.1056/NEJMoa035700 
386

387

[9] Choi HK, Atkinson K, Karlson EW, Willett W, Curhan G. Alcohol intake and risk of incident gout in men: a prospective study. Lancet 2004;363:1277-1281. doi:10.1016/S0140-6736(04)16000-5

[10] Zykova SN, Storhaug HM, Toft I, Chadban SJ, Jenssen TG, White SL. Crosssectional analysis of nutrition and serum uric acid in two Caucasian cohorts: the AusDiab Study and the Tromsø study. Nutr J 2015;14:49. doi:10.1186/s12937-015$0032-1$

[11] Tsai YT, Liu JP, Tu YK, Lee MS, Chen PR, Hsu HC, et al. Relationship between dietary patterns and serum uric acid concentrations among ethnic Chinese adults in Taiwan. Asia Pac J Clin Nutr 2012;21:263-270.

[12] Hu FB. Dietary pattern analysis: a new direction in nutritional epidemiology. Curr Opin Lipidol 2002;13:3-9. doi:10.1097/00041433-200202000-00002

[13] Zhang M, Chang H, Gao Y, Wang X, Xu W, Liu D, et al. Major dietary patterns and risk of asymptomatic hyperuricemia in Chinese adults. J Nutr Sci Vitaminol (Tokyo) 2012;58:339-345. doi:10.3177/jnsv.58.339

[14] Rasheed H, Stamp LK, Dalbeth N, Merriman TR. Interaction of the GCKR and A1CF loci with alcohol consumption to influence the risk of gout. Arthritis Res Ther 2017;19:161. doi:10.1186/s13075-017-1369-y

[15] Batt C, Phipps-Green AJ, Black MA, Cadzow M, Merriman ME, Topless R, et al. Sugar-sweetened beverage consumption: a risk factor for prevalent gout with SLC2A9 genotype-specific effects on serum urate and risk of gout. Ann Rheum Dis 2014;73:2101-6. doi:10.1136/annrheumdis-2013-203600 
408

409

410

411

412

413

414

415

416

417

418

419

420

421

422

423

424

425

426

427

428

429

[16] Roseline YW, Shidoji Y, Hon WM, Masaki M. Association and interaction effect between VEGF receptor-2 (VEGFR-2) gene polymorphisms and dietary pattern on blood uric acid in Malays and Indians. Malays J Nutr 2012;18:307-317.

[17] Liu X, Huang S, Xu W, Zhou A, Li H, Zhang R, et al. Association of dietary patterns and hyperuricemia: a cross-sectional study of the Yi ethnic group in China. Food Nutr Res 2018;62. doi:10.29219/fnr.v62.1380

[18] Zhou AJ, Pan Q, Li AL, Qie YL, Wang QX, Gong Y. Predictive value of obesity and metabolism indexes for hyperuricemia among rural adult Yi residents in Liangshan region. Chinese J Public Health 2015;31:1646-50.

[19] Huang S, Liu X, Li H, Xu W, Jia H. Sex difference in the association of serum uric acid with metabolic syndrome and its components: a cross-sectional study in a Chinese Yi population. Postgrad Med 2017;129:828-33. doi:10.1080/00325481.2017.1372034 [20] Newby PK, Tucker KL. Empirically derived eating patterns using factor or cluster analysis: a review. Nutr Rev 2004;62:177-203. doi:10.1301/nr.2004.may.177-203

[21] Rothman KJ. Epidemiology: An introduction. New York, Oxford University Press, 2002, p.168-180.

[22] Andersson T, Alfredsson L, Källberg H, Zdravkovic S, Ahlbom A. Calculating measures of biological interaction. Eur J Epidemiol 2005;20:575-579. doi:10.1007/s10654-005-7835-x

[23] He F, Wang LL, Yu XL. Dietary patterns associated hyperuricemia among Chinese aged 45 to 59 years: An observational study. Medicine 2017;96:e9248. doi:10.1097/MD.0000000000009248 
430

431

432

433

434

435

436

437

438

439

440

441

442

443

444

445

446

447

448

449

450

451

[24] Shu L, Zheng PF, Zhang XY, Si CJ, Yu XL, Gao W, et al. Association between

Dietary Patterns and the Indicators of Obesity among Chinese: A Cross-Sectional Study.

Nutrients 2015;7:7995-8009. doi:10.3390/nu7095376

[25] Köttgen A, Albrecht E, Teumer A, Vitart V, Krumsiek J, Hundertmark C, et al.

Genome-wide association analyses identify 18 new loci associated with serum urate concentrations. Nat Genet 2013;45:145-154. doi:10.1038/ng.2500

[26] Stiburkova B, Pavelcova K, Pavlikova M, Ješina P, Pavelka K. The impact of dysfunctional variants of ABCG2 on hyperuricemia and gout in pediatric-onset patients. Arthritis Res Ther 2019;21:77. doi:10.1186/s13075-019-1860-8

[27] Dehghan A, Köttgen A, Yang Q, Hwang SJ, Kao WL, Rivadeneira F, et al. Association of three genetic loci with uric acid concentration and risk of gout: a genome-wide association study. Lancet 2008;372:1953-1961. doi:10.1016/S01406736(08)61343-4

[28] Rivera-Paredez B, Macías-Kauffer L, Fernandez-Lopez JC, Villalobos-Comparán M, Martinez-Aguilar MM, de la Cruz-Montoya A, et al. Influence of Genetic and NonGenetic Risk Factors for Serum Uric Acid Levels and Hyperuricemia in Mexicans. Nutrients. 2019;11:1336. doi:10.3390/nu11061336

[29] Nakayama A, Matsuo H, Nakaoka H, Nakamura T, Nakashima H, Takada Y, et al. Common dysfunctional variants of ABCG2 have stronger impact on hyperuricemia progression than typical environmental risk factors. Sci Rep 2014;4:5227. doi: $10.1038 /$ srep05227

[30] Yang B, Mo Z, Wu C, Yang H, Yang X, He Y, et al. A genome-wide association 
452

453

454

455

456

457

458

459

460

461

462

463

464

465

466

467

468

469

470

471

472

study identifies common variants influencing serum uric acid concentrations in a

Chinese population. BMC Med Genomics 2014;7:10. doi:10.1186/1755-8794-7-10

[31] Döring A, Gieger C, Mehta D, Gohlke H, Prokisch H, Coassin S, et al. SLC2A9

influences uric acid concentrations with pronounced sex-specific effects. Nat Genet 2008;40:430-436. doi:10.1038/ng.107

[32] Roseline YW, Shidoji Y, Hon WM, et al. Association and interaction effect between VEGF receptor-2 (VEGFR-2) gene polymorphisms and dietary pattern on blood uric acid in Malays and Indians. Malays J Nutr 2012,18:307-317.

[33] Ching YK, Chin YS, Appukutty M, Ramanchadran V, Yu CY, Ang GY, et al.

Interaction of Dietary Linoleic Acid and $\alpha$-Linolenic Acids with rs 174547

in FADS1 Gene on Metabolic Syndrome Components among Vegetarians. Nutrients 2019;11:1686. doi:10.3390/nu11071686

[34] Wang T, Xu M, Bi Y, Ning G. Interplay between diet and genetic susceptibility in obesity and related traits. Front Med 2018;12:601-607. doi:10.1007/s11684-0180648-6

[35] Zhang X, Meng Q, Feng J, Liao H, Shi R, Shi D, et al. The prevalence of hyperuricemia and its correlates in Ganzi Tibetan Autonomous Prefecture, Sichuan Province, China. Lipids Health Dis 2018;17:235. doi:10.1186/s12944-018-0882-6 
Tables

Table 1 Socio-demographic and clinical characteristics of participants

\begin{tabular}{|c|c|c|c|}
\hline \multirow[t]{2}{*}{ Variables } & \multirow{2}{*}{$\begin{array}{c}\text { Participants with HUA } \\
n=710\end{array}$} & \multirow{2}{*}{$\begin{array}{c}\text { Participants without HUA } \\
\qquad n=1,936\end{array}$} & \multirow[t]{2}{*}{$P$ value } \\
\hline & & & \\
\hline Age (years) & $42.70 \pm 15.06$ & $44.62 \pm 14.09$ & 0.003 \\
\hline Gender $(\%)$ & & & $<0.001$ \\
\hline Male & $504(71.0)$ & $941(48.6)$ & \\
\hline Female & $206(29.0)$ & 995 (51.4) & \\
\hline Ethnic groups (\%) & & & $<0.001$ \\
\hline Han & $351(49.4)$ & $687(35.5)$ & \\
\hline $\mathrm{Yi}$ & $359(50.6)$ & $1249(64.5)$ & \\
\hline Education (\%) & & & $<0.001$ \\
\hline$\leq$ primary school & $276(38.9)$ & $1142(59.0)$ & \\
\hline$\geq$ junior high school & $434(61.1)$ & $794(41.0)$ & \\
\hline Occupation (\%) & & & $<0.001$ \\
\hline Farmer & $251(35.6)$ & $1046(54.3)$ & \\
\hline Non-farmer & $454(64.4)$ & $882(45.7)$ & \\
\hline Waistline $(\mathrm{cm})$ & $84.53 \pm 10.15$ & $78.49 \pm 9.21$ & $<0.001$ \\
\hline WHR & $0.89 \pm 0.06$ & $0.86 \pm 0.06$ & $<0.001$ \\
\hline $\mathrm{BMI}\left(\mathrm{Kg} / \mathrm{m}^{2}\right)$ & $24.21 \pm 3.60$ & $22.43 \pm 3.11$ & $<0.001$ \\
\hline SBP (mmHg) & $131.94 \pm 19.49$ & $126.45 \pm 18.57$ & $<0.001$ \\
\hline $\mathrm{DBP}(\mathrm{mmHg})$ & $80.91 \pm 15.82$ & $77.13 \pm 13.97$ & $<0.001$ \\
\hline SUA $(\mu \mathrm{mol} / \mathrm{L})$ & $466.40 \pm 72.71$ & $306.27 \pm 60.61$ & $<0.001$ \\
\hline $\mathrm{LDL}(\mathrm{mmol} / \mathrm{L})$ & $3.08 \pm 0.84$ & $2.92 \pm 0.80$ & $<0.001$ \\
\hline HDL (mmol/L) & $1.25 \pm 0.32$ & $1.35 \pm 0.33$ & $<0.001$ \\
\hline GLU (mmol/L) & $5.51 \pm 1.39$ & $5.51 \pm 1.54$ & 0.985 \\
\hline $\mathrm{TC}(\mathrm{mmol} / \mathrm{L})$ & $5.10 \pm 1.02$ & $4.93 \pm 1.01$ & $<0.001$ \\
\hline $\mathrm{TG}(\mathrm{mmol} / \mathrm{L})$ & $2.01 \pm 1.45$ & $1.40 \pm 1.15$ & $<0.001$ \\
\hline
\end{tabular}

475 WHR: waist-hip ratio; SBP: systolic blood pressure; DBP: diastolic blood pressure; GLU: glucose; TC:

476 serum cholesterol; TG: serum triglycerides. LDL-cholesterol: low-density lipoprotein cholesterol; HDL-

477 cholesterol: high-density lipoprotein cholesterol. 
Table 2 Factor loading matrix for the tested dietary patterns

\begin{tabular}{lccc}
\hline Food groups & Meat-based & Plant-based & Local special \\
\hline Viscera & $\mathbf{0 . 5 7 7}$ & - & - \\
Snacks and pastries & $\mathbf{0 . 5 3 9}$ & - & - \\
Fish, shrimp, crab and shellfish & $\mathbf{0 . 5 3 7}$ & - & - \\
Fresh meat & $\mathbf{0 . 5 2 1}$ & - & - \\
Eggs & $\mathbf{0 . 5 0 5}$ & - & - \\
Cereals & $\mathbf{0 . 4 5 7}$ & - & - \\
Grain & $\mathbf{0 . 4 5 1}$ & - & - \\
drinks & $\mathbf{0 . 3 5 7}$ & - & - \\
Mushrooms and algae & - & $\mathbf{0 . 7 1 6}$ & - \\
Beans and their products & - & $\mathbf{0 . 6 4 0}$ & - \\
Nuts & - & $\mathbf{0 . 5 2 0}$ & - \\
Fruits & - & $\mathbf{0 . 5 0 3}$ & - \\
Vegetables & - & $\mathbf{0 . 4 8 7}$ & - \\
Milk and dairy products & - & $\mathbf{0 . 3 9 6}$ & $\mathbf{0 . 7 2 7}$ \\
Marinated and smoked meat & - & - & $\mathbf{0 . 5 8 8}$ \\
Grease & - & - & - \\
Alcoholic beverages & - & 12.542 & 8.686 \\
Contribution rate $(\%)$ & 12.640 & 25.182 & \\
The cumulative contribution rate & 12.640 & & -869 \\
(\%) & & & - \\
\hline
\end{tabular}

Factor loading $>0.30$ are listed. 
Table 3 Association between dietary patterns and HUA

\begin{tabular}{|c|c|c|c|c|c|}
\hline \multirow{2}{*}{$\begin{array}{l}\text { dietary } \\
\text { pattern }\end{array}$} & \multirow{2}{*}{ Quartile } & \multicolumn{2}{|c|}{ Crude model } & \multicolumn{2}{|c|}{ Adjusted model } \\
\hline & & OR $(95 \% \mathrm{CI})$ & $P$ value & OR $(95 \% \mathrm{CI})$ & $P$ value \\
\hline \multirow{4}{*}{$\begin{array}{l}\text { Meat- } \\
\text { based }\end{array}$} & $\mathrm{Q}_{1}$ & 1.00 & & 1.00 & \\
\hline & $\mathrm{Q}_{2}$ & $1.446(1.118 \sim 1.870)$ & 0.005 & $1.307(1.003 \sim 1.702)$ & 0.048 \\
\hline & $\mathrm{Q}_{3}$ & $1.644(1.275 \sim 2.119)$ & $<0.001$ & $1.288(0.984 \sim 1.685)$ & 0.065 \\
\hline & $\mathrm{Q}_{4}$ & $1.878(1.461 \sim 2.415)$ & $<0.001$ & $1.385(1.052 \sim 1.823)$ & 0.020 \\
\hline \multirow[t]{4}{*}{$\begin{array}{l}\text { Plant- } \\
\text { based }\end{array}$} & $\mathrm{Q}_{1}$ & 1.00 & & 1.00 & \\
\hline & $\mathrm{Q}_{2}$ & $1.119(0.875 \sim 1.432)$ & 0.371 & $1.086(0.842 \sim 1.401)$ & 0.525 \\
\hline & $\mathrm{Q}_{3}$ & $1.076(0.841 \sim 1.379)$ & 0.559 & $0.960(0.741 \sim 1.245)$ & 0.760 \\
\hline & $\mathrm{Q}_{4}$ & $1.268(0.994 \sim 1.617)$ & 0.056 & $1.080(0.831 \sim 1.404)$ & 0.566 \\
\hline \multirow{4}{*}{$\begin{array}{l}\text { Local } \\
\text { special }\end{array}$} & $\mathrm{Q}_{1}$ & 1.00 & & 1.00 & \\
\hline & $\mathrm{Q}_{2}$ & $0.932(0.735 \sim 1.181)$ & 0.558 & $1.069(0.837 \sim 1.365)$ & 0.592 \\
\hline & $\mathrm{Q}_{3}$ & $0.871(0.686 \sim 1.105)$ & 0.255 & $1.111(0.864 \sim 1.429)$ & 0.412 \\
\hline & $\mathrm{Q}_{4}$ & $0.635(0.495 \sim 0.815)$ & $<0.001$ & $0.865(0.662 \sim 1.131)$ & 0.289 \\
\hline
\end{tabular}

491

492

493

494

495

496

497

498

499

500

501

502

Crude model: no adjustments are made. Adjusted model: adjusted for age (continuous), gender (male/female), and ethnicity.

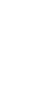


Table 4 Frequency distribution of $A B C G 2$ rs2231142 and SLC2A9 rs11722228 loci

\begin{tabular}{ccccc}
\hline SNP sites & Genotype/Allele & HUA group & Non-HUA group & P value \\
\hline rs2231142 & G:G & $374(52.7)$ & $1349(69.7)$ & $<\mathbf{0 . 0 0 1}$ \\
& G:T & $278(392)$ & $528(27.3)$ & \\
T:T & $58(8.2)$ & $59(3.0)$ & \\
G & $1026(72.3)$ & $3226(83.3)$ & $<\mathbf{0 . 0 0 1}$ \\
T & $394(27.7)$ & $646(16.7)$ & \\
rs11722228 & C:C & $301(42.4)$ & $904(46.7)$ & 0.074 \\
C:T & $328(46.2)$ & $854(44.1)$ & \\
T:T & $81(11.4)$ & $178(9.2)$ & \\
C & $930(65.5)$ & $2662(68.8)$ & 0.025 \\
& T & $490(34.5)$ & $1210(31.2)$ & \\
\hline
\end{tabular}

505

506

507

508

509

510

511

512

513

514

515

516 
Table 5 Additive interaction of dietary patterns with ABCG2 rs2231142 and SLC2A9

519 rs11722228 loci

\begin{tabular}{lllll}
\hline SNP site & Dietary patterns & RERI & AP & S \\
\hline rs2231142 & Meat-based & $0.482(0.012 \sim 0.976)$ & $0.203(0.033 \sim 0.374)$ & $1.544(1.012 \sim 2.355)$ \\
& Plant-based & $0.049(-0.421 \sim 0.519)$ & $0.022(-0.183 \sim 0.227)$ & $1.041(0.713 \sim 1.520)$ \\
& Local special & $-0.140(-0.496 \sim 0.215)$ & $-0.099(-0.353 \sim 0.156)$ & $0.750(0.367 \sim 1.534)$ \\
rs11722228 & Meat-based & $0.024(-0.551 \sim 0.600)$ & $0.009(-0.208 \sim 0.226)$ & $1.015(0.713 \sim 1.446)$ \\
& Plant-based & $-0.033(-0.367 \sim 0.302)$ & $-0.022(-0.244 \sim 0.201)$ & $0.941(0.501 \sim 1.764)$ \\
& Local special & $-0.052(-0.298 \sim 0.194)$ & $-0.055(-0.315 \sim 0.205)$ & $14.979(0.000 \sim 33.426)$ \\
\hline
\end{tabular}

520

521

522

523

524

525

526

527

528

529

530

531

532

533

534

535 


\section{Figure Legends}

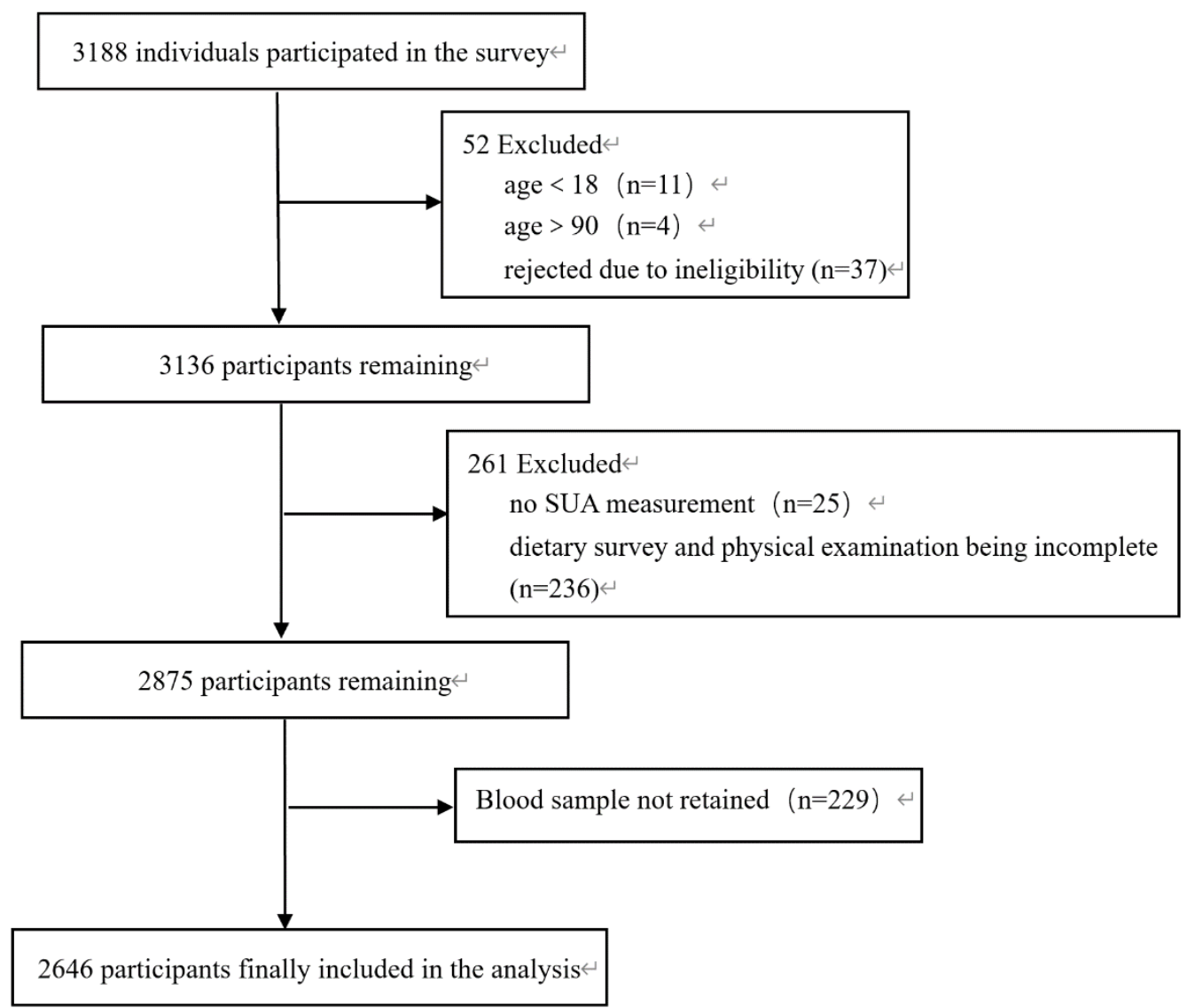

537

538 Figure 1 Participant flowchart

539

540

541 

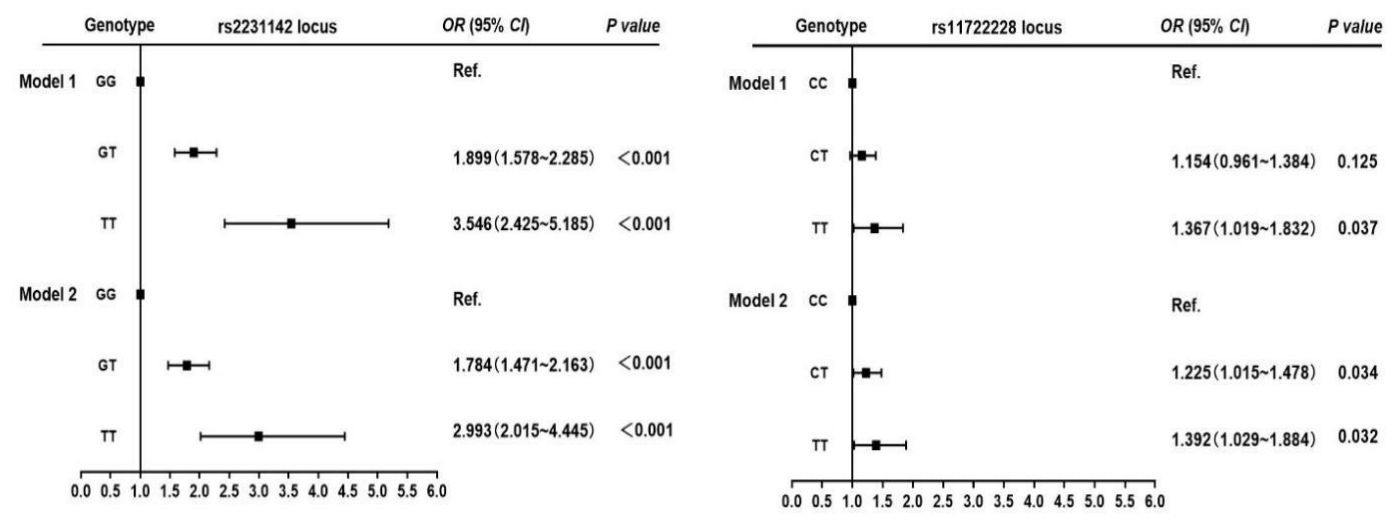

542

543 Model 1: not adjusted are made. Model 2: adjusted for age, gender, and ethnicity.

544 Figure 2 The relationship between $A B C G 2$ rs2231142 and SLC2A9 rs11722228 loci

545 with HUA

546

547

548

549

550

551

552

553

554

555

556 


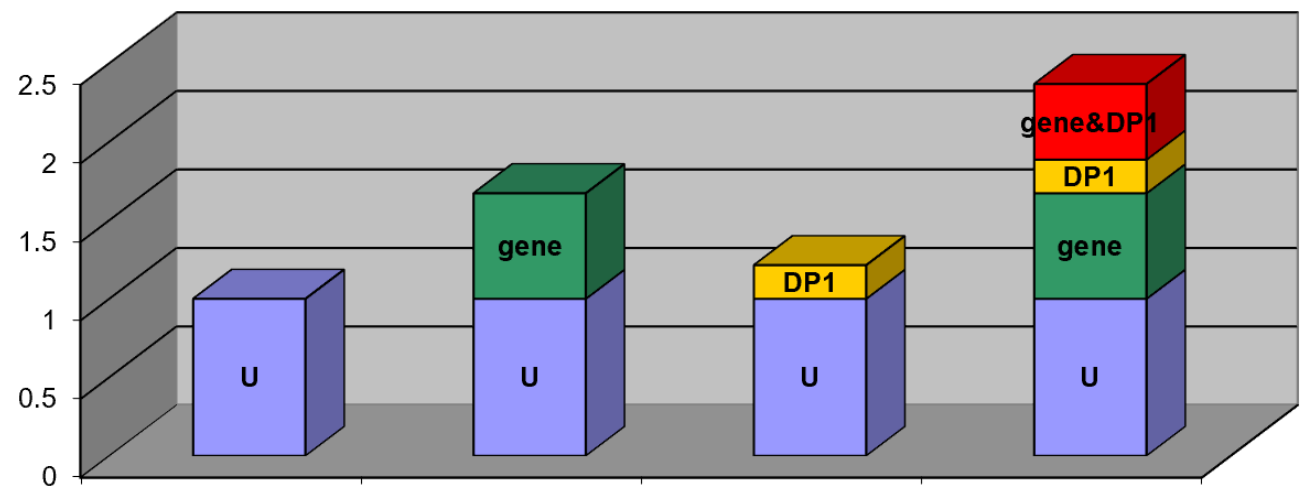

559 U: Baseline; gene: $A B C G 2$ rs2231142locus; DP1: Dietary pattern 1 (Meat-based pattern); gene\&DP1: Additive 560 interaction between the 'Meat-based' pattern and $A B C G 2$ rs2231142 locus.

561 Figure 3 Additive interaction between the 'Meat-based' pattern and $A B C G 2$ rs2231142 locus 


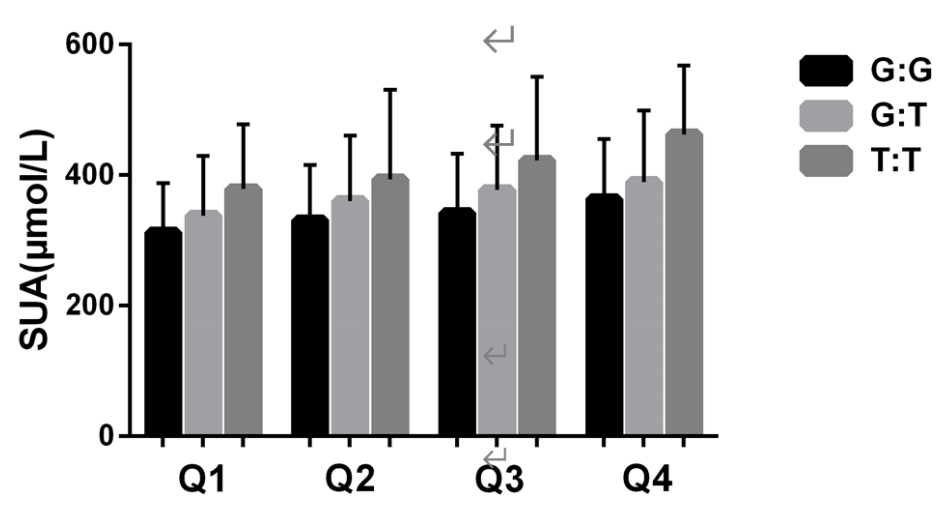

578 Figure 4 The SUA level of $A B C G 2$ rs2231142 locus in 'Meat-based' pattern quartile 